\title{
A Review on D 614G Mutation with Bangladesh Scenario
}

\author{
Arifa Akram ${ }^{*}$ (1), Mohammad Jahidur Rahman Khan², Md. Bayzid Bin Monir ${ }^{3}$, \\ Md. Reaz Uddin Chowdhury4, Mahmuda Yeasmin'1, Md. Maruf Ahmed Molla1, \\ Tasnim Nafisa ${ }^{1}$
}

${ }^{1}$ Department of Virology, National Institute of Laboratory Medicine and Referral Center, Dhaka, Bangladesh

${ }^{2}$ Department of Microbiology, Shaheed Suhrawardy Medical College, Dhaka, Bangladesh

${ }^{3}$ Department of Microbiology, National Institute of Laboratory Medicine and Referral Center, Dhaka, Bangladesh

${ }^{4}$ Abdul Malek Ukil Medical College, Noakhali, Bangladesh

Email: ^drbarna43@gmail.com

How to cite this paper: Akram, A., Khan, M.J.R., Monir, Md.B.B., Chowdhury, Md.R.U., Yeasmin, M., Molla, Md.M.A. and Nafisa, T. (2020) A Review on D 614G Mutation with Bangladesh Scenario. Advances in Infectious Diseases, 10, 249-255.

https://doi.org/10.4236/aid.2020.103024

Received: November 15, 2020

Accepted: December 15, 2020

Published: December 18, 2020

Copyright $\odot 2020$ by author(s) and Scientific Research Publishing Inc. This work is licensed under the Creative Commons Attribution International License (CC BY 4.0)

http://creativecommons.org/licenses/by/4.0/

\section{(c) (i) Open Access}

\begin{abstract}
With the COVID-19 pandemic, disparities between the infection rate and death rate in different countries become a major concern. In some countries, lower mortality rate compared to others can be explained by better testing capacity and intensive care facilities. Complete SARS-CoV-2 genome sequences from different countries of the world are continually submitted to Global Initiative for Sharing All Influenza Data using Next Generation Sequencing method. A SARS-CoV-2 variant with a D 614G Mutation in the spike (S) protein has become the most dominant form in the global pandemic. There are a number of ongoing studies trying to relate this mutation with the infectivity, mortality, transmissibility of the virus and its impact on vaccine development. This review aims to accumulate the major findings from some of these studies and focus its future implication. Some studies suggested D 614G strain has increased binding capacity, it affects more cells at a faster rate, so has a high transmissibility. Patients infected with this strain were found with high viral load. But still now there is no such evidence that this strain produces more severe disease as well as increased mortality. The structural change of spike protein produced by D $614 \mathrm{G}$ mutation was minor and did not hamper the vaccine efficacy. Some studies showed antibodies produced against D614 strain can neutralize G614 strain and vice versa. Whenever a mutation occurs in spike protein there are always chances of affecting the infectivity, transmissibility, vaccine efficacy. Therefore, more studies are required to find out the overall effect of D 614G mutation.
\end{abstract}

\section{Keywords}

Coronavirus, D 614G Mutation, SARS-CoV-2, Spike Protein, Global Initiative 
for Sharing All Influenza Data (GISAID)

\section{Background}

The rapid spread of novel SARS-CoV-2 across the world has become a significant threat to civilization [1]. By June 17, 2020, the World Health Organization (WHO) reported that 8.06 million people worldwide had been infected with SARSCoV-2, and 440,290 individuals died of COVID-19. This pandemic has affected international social and economic activities markedly. To facilitate diagnosis, molecular epidemiologic source tracking, and for vaccines and therapeutic strategies, RNA genome was sequenced rapidly [2]. Humans have no previous immunological experience with the novel virus and for that creates severe infection and disease. SARS-CoV-2 is highly transmissible: basic reproduction number, R0, estimates vary between 2.2 and 3.9. Mortality vary regionally between $0.8 \%$ and 14.5\% (mortality analyses, Johns Hopkins University of Medicine) [3].

Coronaviruses have genetic proofreading mechanisms, and SARS-CoV-2 sequence diversity is very low. Still, natural selection can act upon rare but favorable mutations [3].

Currently, complete SARS-CoV-2 sequences using NGS technology from all the countries are being submitted at GISAID [4]. To understand their transmission, evolutionary and genetic relationships through phylogeny, these sequences are being scrutinized in detail. Though, it is unclear whether the SARSCoV-2 genetic mutations have impact on transmissibility and/or virulent property [1].

\section{What Is D 614G Mutation?}

D 614G Mutation is characterized by aspartic acid to glycine shift at the amino acid position 614 and it is permanent change. The initial D614 is now the G614 variant. It may occur due to environmental factors (UV radiation), or due to an error during DNA replication process. Missense, nonsense, insertion, deletion, duplication, frame shift, and repeat are some types of mutation [5].

Overall, viruses can undergo frequent genetic mutations for some reasons, such as natural selection and random genetic drifts. As these factors can work sequentially, it is difficult to identify when a viral mutation becomes more prevalent.

D 614G mutation in the viral spike protein occurred at the initial stage of the pandemic in the case of a novel coronavirus. To detect the causative factors responsible for the rapid emergence of G 614 containing coronavirus, scientists have monitored all coronavirus genome sequencing data that are available globally at the Global Initiative for Sharing All Influenza Data (GISAID) database [5].

D 614G strain by itself is more deadly because its binding capacity in enhanced, it is able to affect more host cell, tissues and also organ faster [6]. 


\section{D 614G Mutation and Infectivity}

There is no evidence that infection with SARS-CoV-2 containing the G 614 variant will lead to more severe disease at this moment. By examining clinical data from 999 COVID-19 cases diagnosed in the United Kingdom, Korber et al. (2020) found that patients infected with viruses containing G 614 had higher levels of virus RNA, though they did not find a difference in hospitalization outcomes [3]. These observations are supported by two independent studies: 175 COVID-19 patients from Seattle, WA (Wagner et al., 2020) and 88 COVID-19 patients from Chicago, IL (Lorenzo-Redondo et al., 2020) in the United States [7] [8]. Viral load and disease severity are not always correlated. The current evidence suggests that D 614G is not so much important for COVID-19 than other risk factors, such as age or comorbidities [9].

Another published study in Cell out of the WHO Collaborating Center in China demonstrated the same. "This dominant [G614] strain could effectively infect the four cell lines tested, being 10-fold more infectious than the original Wuhan-1 strain," the study authors wrote. Another research review published in Cell in July too, where scientists suggest, but not prove, increased viral transmissibility [10].

\section{D 614G Mutation and Mortality}

Although there is no evidence showing that D 614G mutation is associated with increased COVID19 severity, a recent study using a phylogenetic tree of more than 4000 coronavirus genomes has claimed that viruses containing D 614G mutation are more virulent, and thus, are associated with higher Disease related mortality [5].

National University of Singapore and president-elect of the International Society of Infectious Diseases, said evidence suggests the proliferation of the D 614G mutation in some parts of the world has coincided with a drop in death rates, suggesting it is less lethal [11].

While Korber et al. found an increased viral load in patients infected with the G614 variant, there was no significant association with disease severity [3]. Two other preprints-one investigating 175 Covid-19 patients from Seattle and the other 88 from Chicago-also did not find any relationship between the G614 variant and clinical outcomes [12].

\section{D 614G Mutation and Vaccine}

As spike protein is located on the outer surface of the virus, it's close to host immune system. Thus, spike D 614G mutation may play vital role in modulating the viral ability to escape vaccine induced immune responses. However, some evidence suggests that the possibility of D $614 \mathrm{G}$ mutation affecting the vaccine efficacy is very less. As this mutation is not in the receptor binding domain of the spike protein, it can't affect the ability of the domain which induce host immune responses, and it is essential for antibody mediated neutralization of the 
virus [5].

The RBD binds to the ACE2 receptor on human cells; it is also the main target of the immune system. However, it is showed that the D $614 \mathrm{G}$ mutation produces a minor change in the spike protein structure. This mutation likely enables the spike protein to prime itself in such a way that the RBD can bind the ACE2 receptor more efficiently. Prof. Grubaugh and others. Their review also cited three studies showing that antibodies built from natural D614 and G614 infections can cross-neutralize-meaning that antibodies made against D614 could work for G614, and vice-versa [12].

D 614G mutation has no effect on vaccine efficacy and many trials of ongoing vaccines are developed against the Receptor binding domain. Study showed that convalescent sera obtained people infected with D614 containing viruses have been found to neutralize viruses containing G614, and vice versa which indicates that D 614G mutation does not alter antibody mediate immune responses [5].

\section{Bangladesh Scenario}

D $614 \mathrm{G}$ in the spike glycoprotein is also predominant in Bangladesh originated SARS-CoV-2 genome, which should be of urgent concern considering the dominance of this mutation globally since early February in Europe [13].

From Bangladesh, the very first complete genome of SARS-CoV-2 (hCoV-19/ Bangladesh/CHRF_nCOV19_0001/2020 under GISAID Accession No. EPI/ISL/ 437912) was published by the Child Health Research Foundation (CHRF) on May 12, 2020. The first complete sequence data is of the virus strain sampled from 22 years aged female patient on 18 April 2020, although not mentioned, possibly the person had no recent travel history [14] [15].

Another group of scientists of Bangladesh compared 207 of SARS-CoV-2 genomes reported from different parts of Bangladesh and their comparison with 467 globally reported sequences to understand the origin of viruses, possible patterns of mutations, availability of unique mutations, and their apparent impact on pathogenicity of the virus in victims of Bangladeshi population. Phylogenetic analyses indicate that in Bangladesh, SARS-CoV-2 viruses might arrive through infected travelers from European countries, and the GR clade was found as predominant in this region. It is observed that $95.6 \%$ of genomes from Bangladesh have D 614G mutation, which is also dominant in the world [14]. They found 2602 mutations including 1602 missense mutations, 612 synonymous mutations, 36 insertions and deletions with 352 other mutations types. In line with the global trend, D 614G mutation in spike glycoprotein was predominantly high (95.6\%) in Bangladeshi isolates. Interestingly, we found the average number of mutations in ORF1ab, S, ORF3a, $\mathrm{M}$ and $\mathrm{N}$ of genomes, having nucleotide shift at G614 ( $\mathrm{n}=459)$, were significantly higher $(\mathrm{p} \leq 0.001)$ than those having mutation at D614 $(\mathrm{n}=215)$. Previously reported frequent mutations such as P4715L, D 614G, R203K, G204R and I300F were also prevalent in Bangladeshi isolates. Additionally, 87 unique amino acid changes were revealed and were categorized as 
originating from different cities of Bangladesh. The analyses would increase our understanding of variations in virus genomes circulating in Bangladesh and elsewhere and help develop novel therapeutic targets against SARS-CoV-2 [16]. This correlation indicates that the genomes containing D $614 \mathrm{G}$ mutation are more prone to bear other mutations which may facilitate the notion that the link of this mutation with the transmission and pathogenesis of SARS-CoV-2 [14].

To investigate the genetic diversity, a total of 263 genomes of SARS-CoV-2 strains were sequenced by genomic Research Lab, BCSIR, A total of 737 nucleotide mutations located along the entire genome resulting in non-synonymous 358 amino acid substitutions in 25 different proteins were detected. The nucleotide mutation rate is estimated to be 24.6 substitutions per year. The highest nucleotide mutations were observed at 112 positions of non-structural protein papain-like protease (nsp3), which led to the 62 non-synonymous amino acid substitutions. Among the structural proteins, the highest mutations were observed at 101 positions of spike proteins resulting in 53 non-synonymous amino acid substitution. The only dominated variant "G614" (due to the change of aspartic acid at 614 number to glycine in spike protein) found in $100 \%$ of cases is circulating across the country with co-evolving other variants including L323 (100\%) in RNA dependent RNA polymerase (RdRp), K203 and R204 (93.5\%) in nucleocapsid, and F120 (83\%) in NSP2. Global mutation event per sample is 7.23

Table 1. Showing different mutations found in Bangladesh which is published in different articles.

Serial Articles Findings

1 Saha S et al, 2020. Complete genome sequence of a novel 9 coronavirus (SARS-CoV-2) isolate from Bangladesh. Microbiol Resour Announc 9: e00568-20.

9 mutations were observed in CHRF_nCoV19_0001, compared to the https://doi.org/10.1128/MRA.00568-20. from December 2019. These mutations included the spike protein D614G mutation that is enriched in recent SARS-CoV-2 isolates [15]. Average number of mutations in ORF1ab, S, ORF3a, M and $\mathrm{N}$ of genomes, Bangladeshi SARS-CoV-2 Genomes doi: https://doi.org/10.1101/2020.08.25.267658 having nucleotide shift at G614 $(\mathrm{n}=459)$, were significantly higher $(\mathrm{p} \leq 0.001)$ than those having mutation at D614 $(n=215)$. Previously reported frequent mutations such as P4715L, D614G, R203K, G204R and I300F were also prevalent in Bangladeshi isolates. Additionally, 87 unique amino acid changes were revealed [16]. Non-synonymous Mutation, And Genomic Phylogeny of non-structural protein papain-like protease (nsp3), which led to the 62 263 SARS-CoV-2 Sequenced By Genomic Research Lab, non-synonymous amino acid substitutions. Among the structural proteins, BCSIR. Available at: the highest mutations were observed at 101 positions of spike proteins file:///E:/papers/d\%20164\%20g/Epidemiological\%20Repo rt-BCSIR_Bangladesh_2020\%20v1.1.pdf resulting in 53 non-synonymous amino acid substitution. The only dominated variant "G614" (due to the change of aspartic acid at 614 number to glycine in spike protein) found in $100 \%$ of cases is circulating across the country with co-evolving other variants including L323 (100\%) in RNA dependent RNA polymerase (RdRp), K203 and R204 (93.5\%) in nucleocapsid, and F120 (83\%) in NSP2 [17].

Highest mutations at 36 different positions have identified in spike proteins. A total of 9 mutations at spike proteins were found unique in relative to the global mutations including mutations at position 516 in the boundary of the ACE2 binding region of the spike protein [18]. 
while, it is 12.6 in Bangladesh, indicating SARS-CoV changing rapidly in Bangladesh than the rest of the world [17] (Table 1).

\section{Conclusion}

The number of confirmed COVID-19 is still rising and no sign of waning in the near future. This pandemic will be long, until effective anti-viral drugs or vaccines are available. Still now, overall genetic variation of SARS-CoV-2 is low, and SARS$\mathrm{CoV}-2$ viruses that are currently circulating establish a similar viral population. So far, the D 614G strain did not possess any major threat regarding infectivity, transmissibility or antigenicity, drug and vaccine developers should be alert for the emergence of new variants like D 614G strain. Further studies on the currently circulating virus are also important in pandemic planning and the efficiency of pandemic response. Extensive gene sequencing has to be performed in this regard, from different part of the world.

\section{Ethical Approval}

The study was approved by the NILMRC Ethics Committee.

\section{Conflicts of Interest}

The authors declare no conflicts of interest regarding the publication of this paper.

\section{References}

[1] Eaaswarkhanth, M., Al Madhoun, A. and Al-Mulla, F. (2020) Could the D614 G Substitution in the SARS-CoV-2 Spike (S) Protein Be Associated with Higher COVID-19 Mortality? International Journal of Infectious Diseases, 96, 459-460. https://doi.org/10.1016/j.ijid.2020.05.071

[2] Hu, J., He, C.L., Gao, Q., Zhang, G.J., Cao, X.X., Long, Q.X., Deng, H.J., Huang, L.Y., Chen, J., Wang, K. and Tang, N. (2020) The D 614G Mutation of SARS-CoV-2 Spike Protein Enhances Viral Infectivity.

[3] Korber, B., Fischer, W.M., Gnanakaran, S., Yoon, H., Theiler, J., Abfalterer, W., Hengartner, N., Giorgi, E.E., Bhattacharya, T., Foley, B. and Hastie, K.M. (2020) Tracking Changes in SARS-CoV-2 Spike: Evidence that D 614G Increases Infectivity of the COVID-19 Virus. Cell, 182, 812-827.

[4] Shu, Y. and McCauley, J. (2017) GISAID: Global Initiative on Sharing All Influenza Data-From Vision to Reality. Eurosurveillance, 22, 30494.

https://doi.org/10.2807/1560-7917.ES.2017.22.13.30494

[5] Dr. Sanchari Sinha Dutta, Ph.D. D614 G Mutation. https://www.news-medical.net/health/D614G-Mutation.aspx

[6] What the D614G Mutation Means for SARS-CoV-2 Vaccines. Genomics. https://www.technologynetworks.com/genomics/news/what-the-d614g-mutation-m eans-for-sars-cov-2-vaccines-342786

[7] Wagner, C., Roychoudhury, P., Hadfield, J., Hodcroft, E.B., Lee, J., Moncla, L.H., Müller, N.F., Behrens, C., Huang, M.L., Mathias, P. and Pepper, G. (2020) Comparing Viral Load and Clinical Outcomes in Washington State across D 614G Mutation in Spike Protein of SARS-CoV-2. https://github.com/blab/ncov-wa-d614g 
[8] Lorenzo-Redondo, R., Nam, H.H., Roberts, S.C., Simons, L.M., Jennings, L.J., Qi, C., Achenbach, C.J., Hauser, A.R., Ison, M.G., Hultquist, J.F. and Ozer, E.A. (2020) A Unique Clade of SARS-CoV-2 Viruses Is Associated with Lower Viral Loads in Patient Upper Airways. https://doi.org/10.1101/2020.05.19.20107144

[9] Grubaugh, N.D., Hanage, W.P. and Rasmussen, A.L. (2020) Making Sense of Mutation: What D 614G Means for the COVID-19 Pandemic Remains Unclear. Cell, 182, 794-795. https://doi.org/10.1016/j.cell.2020.06.040

[10] Yong, S.J. What the D 614G Mutation Means for Covid-19 Spread, Fatality, Treatment, and Vaccine.

https://medium.com/microbial-instincts/what-the-D614G-mutation-means-for-cov id-19-spread-fatality-treatment-and-vaccine-7dda1c066f0d

[11] Mutated Coronavirus May Be Less Deadly, Expert Suggests. Aljazeera, News, Corona Virus Pandemic.

https://www.aljazeera.com/news/2020/08/mutated-coronavirus-deadly-expert-sugg ests-200818040211070.html

[12] What the D 614G Mutation Means for Covid-19 Spread, Fatality, Treatment, and Vaccine.

https://medium.com/microbial-instincts/what-the-D614G-mutation-means-for-cov id-19-spread-fatality-treatment-and-vaccine-7dda1c066f0d

[13] Saha, O., Shatadru, R.N., Rakhi, N.N., Islam, I., Hossain, M.S. and Rahaman, M.M. (2020) Temporal Landscape of Mutation Accumulation in SARS-CoV-2 Genomes from Bangladesh: Possible Implications from the Ongoing Outbreak in Bangladesh.

[14] Ul Alam, A.R., Rafiul Islam, M., Shaminur Rahman, M., Islam, O.K. and Anwar Hossain, M. (2020) Understanding the Possible Origin and Genotyping of First Bangladeshi SARS-CoV-2 Strain. Journal of Medical Virology. https://doi.org/10.31219/osf.io/8j29u

[15] Saha, S., Malaker, R., Sajib, M.S.I., Hasanuzzaman, M., Rahman, H., Ahmed, Z.B., Islam, M.S., Islam, M., Hooda, Y., Ahyong, V., Vanaerschot, M., Batson, J., Hao, S., Kamm, J., Kistler, A., Tato, C.M., DeRisi, J.L. and Saha, S.K. (2020) Complete Genome Sequence of a Novel Coronavirus (SARS-CoV-2) Isolate from Bangladesh. Microbiology Resource Announcements, 9, e00568-20. https://doi.org/10.1128/MRA.00568-20

[16] Hasan, M.M., Das, R., Rasheduzzaman, M., Hussain, M.H., Muzahid, N.H., Salauddin, A., Rumi, M.H., Rashid, S.M., Siddiki, A.Z. and Mannan, A. (2020) Global and Local Mutations in Bangladeshi SARS-CoV-2 Genomes.

[17] Study of Transmission, Mutation Rate, Genetic Variants, Non-Synonymous Mutation, and Genomic Phylogeny of 263 SARS-CoV-2 Sequenced by Genomic Research Lab, BCSIR.

[18] Mahmud, A.S., Taznin, T., Sarkar, M.M., Uzzaman, M.S., Osman, E., Habib, M.A. Akter, S., Banu, T.A., Goswami, B., Jahan, I. and Hossain, M.S. (2020) The Genetic Variants Analysis of Circulating SARS-CoV-2 in Bangladesh. 